EPJ Web of Conferences 59, 01008 (2013)

DOI: $10.1051 /$ epjconf/20135901008

(C) Owned by the authors, published by EDP Sciences, 2013

\title{
Implosion and heating experiments of fast ignition targets by Gekko-XII and LFEX lasers
}

\author{
H. Shiraga ${ }^{1}$ a, S. Fujioka ${ }^{1}$, M. Nakai ${ }^{1}$, T. Watari ${ }^{1}$, H. Nakamura ${ }^{1}$, Y. Arikawa ${ }^{1}$, \\ H. Hosoda ${ }^{1}$, T. Nagai ${ }^{1}$, M. Koga ${ }^{1}$, H. Kikuchi ${ }^{1}$, Y. Ishii ${ }^{1}$, T. Sogo ${ }^{1}$, \\ K. Shigemori ${ }^{1}$, H. Nishimura ${ }^{1}$, Z. Zhang ${ }^{1}$, M. Tanabe ${ }^{1}$, S. Ohira ${ }^{1}$, Y. Fujii ${ }^{1}$, \\ T. Namimoto ${ }^{1}$, Y. Sakawa ${ }^{1}$, O. Maegawa ${ }^{1}$, T. Ozaki ${ }^{2}$, K.A. Tanaka ${ }^{3}$, \\ H. Habara ${ }^{3}$, T. Iwawaki ${ }^{1}$, K. Shimada ${ }^{1}$, M. Key ${ }^{4}$, P. Norreys ${ }^{5}$, J. Pasley ${ }^{6}$, \\ H. Nagatomo ${ }^{1}$, T. Johzaki ${ }^{7}$, A. Sunahara ${ }^{7}$, M. Murakami $^{1}$, H. Sakagami ${ }^{2}$, \\ T. Taguchi ${ }^{8}$, T. Norimatsu ${ }^{1}$, H. Homma ${ }^{1}$, Y. Fujimoto ${ }^{1}$, A. Iwamoto ${ }^{2}$, \\ N. Miyanaga ${ }^{1}$, J. Kawanaka ${ }^{1}$, T. Kanabe ${ }^{9}$, T. Jitsuno ${ }^{1}$, Y. Nakata ${ }^{1}$, \\ K.Tsubakimoto ${ }^{1}$, K. Sueda ${ }^{3}$, R. Kodama ${ }^{3}$, K. Kondo ${ }^{10}$, N. Morio ${ }^{1}$, S. Matsuo ${ }^{1}$, \\ T. Kawasaki ${ }^{1}$, K. Sawai ${ }^{1}$, K. Tsuji ${ }^{1}$, H. Murakami ${ }^{1}$, N. Sarukura ${ }^{1}$, T. Shimizu ${ }^{1}$, \\ K. Mima ${ }^{1}$ and $\mathrm{H}$. Azechi ${ }^{1}$ \\ 1 Institute of Laser Engineering, Osaka University, Suita, Osaka 565-0871, Japan \\ 2 Nationai Institute for Fusion Science, Toki, Gifu 509-5292, Japan \\ 3 Graduate School of Engineering, Osaka University, Suita, Osaka 565-0871, Japan \\ ${ }^{4}$ Lawrence Livermore National Laboratory, Livermore, California 94550, USA \\ ${ }^{5}$ Rutherford Appleton Laboratory, STFC Rutherford Appleton Laboratory, Harwell Oxford, \\ Didcot, Oxfordshire OX11 OQX, UK \\ 6 York Plasma Institute, Department of Physics, University of York, York YO10 5DQ, UK \\ 7 Institute for Laser Technology, Suita, Osaka 565-0871, Japan \\ 8 Faculty of Science and Engineering, Setsunan University, Neyagawa, Osaka 572-8508, \\ Japan \\ 9 Graduate School of Engineering, University of Fukui, Fukui, Fukui 910-8507, Japan \\ 10 Kansai Photon Science Institute, Japan Atomic Energy Agency, Kizu, Kyoto 619-0215, \\ Japan
}

\begin{abstract}
The FIREX-1 project, the goal of which is to demonstrate fuel heating up to $5 \mathrm{keV}$ by fast ignition scheme, has been carried out since 2003 including construction and tuning of LFEX laser and integrated experiments. Implosion and heating experiment of Fast Ignition targets have been performed since 2009 with Gekko-XII and LFEX lasers. A deuterated polystyrene shell target was imploded with the $0.53-\mu \mathrm{m}$ Gekko-XII, and the 1.053- $\mu \mathrm{m}$ beam of the LFEX laser was injected through a gold cone attached to the shell to generate hot electrons to heat the imploded fuel plasma. Pulse contrast ratio of the LFEX beam was significantly improved. Also a variety of plasma diagnostic instruments were developed to be compatible with harsh environment of intense hard $\mathrm{x}$-rays $(\gamma$ rays) and electromagnetic pulses due to the intense LFEX beam on the target. Large background signals around the DD neutron signal in timeof-flight record of neutron detector were found to consist of neutrons via $(\gamma, \mathrm{n})$ reactions and scattered gamma rays. Enhanced neutron yield was confirmed by carefully eliminating such backgrounds. Neutron enhancement up to $3.5 \times 10^{7}$ was observed. Heating efficiency was estimated to be $10-20 \%$ assuming a uniform temperature rise model.
\end{abstract}

\footnotetext{
ae-mail: shiraga@ile.osaka-u.ac.jpr
}

This is an Open Access article distributed under the terms of the Creative Commons Attribution License 2.0, which permits unrestricted use, distribution, and reproduction in any medium, provided the original work is properly cited. 

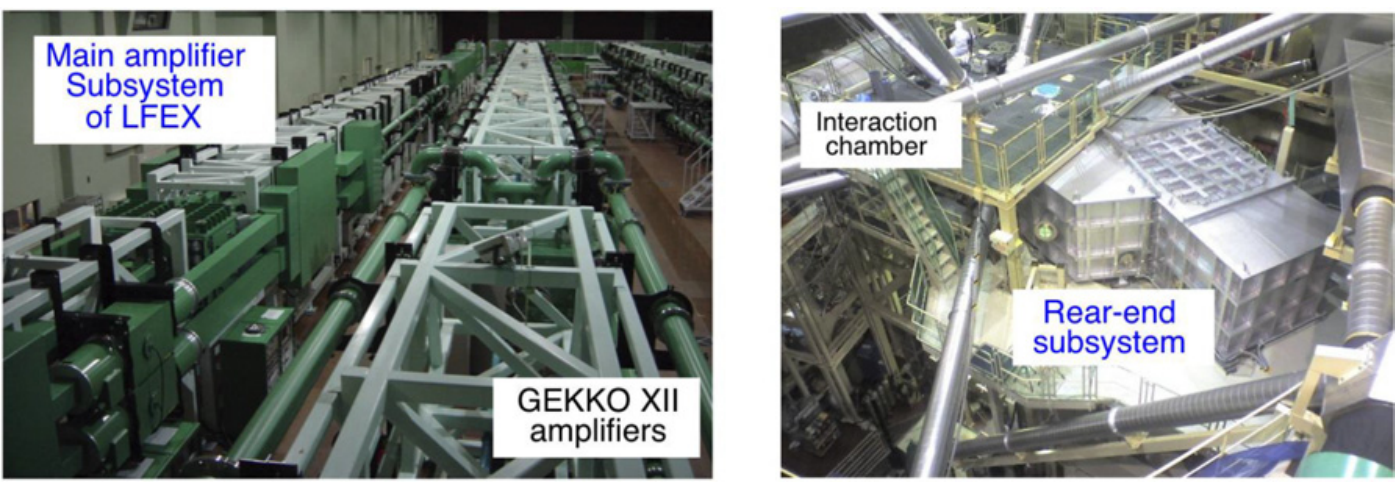

Figure 1. (left) Gekko-XII laser system for implosion of the fuel target, and LFEX laser beam line for Fast Ignition heating. (right) Rear-end subsystem including pulse compressor and focusing optics inside the vacuum chamber, and the interaction chamber.

\section{INTRODUCTION - FIREX PROJECT}

Fast ignition (FI) is a new scheme for laser-driven inertial confinement fusion [1-3]. First, a fuel pellet is imploded by a long-pulse drive laser, and then, a short-pulse high-intensity heating laser is injected to the fuel plasma at the time of maximum fuel $\rho$ R. Since it is not required to form a central hot spark inside the main fuel, the driver energy in FI could be reduced by one order of magnitude. A shell target with a cone for guiding the heating laser was proposed for the FI scheme, and successful FI heating up to $1 \mathrm{keV}$ was achieved with $1 \mathrm{~kJ} \mathrm{PW}$ laser facility [4]. Based on this result, the FIREX-1 project was started since 2003 at the Institute of Laser Engineering, Osaka University [5]. Its goal is to demonstrate fuel heating up to $5 \mathrm{keV}$ with a 10-kJ class new heating laser, LFEX.

Construction of LFEX was started in 2004, and it became partially operational in 2008. In 2009, the first campaign of FI integrated experiment was performed by using Gekko-XII laser for implosion and LFEX laser for heating, in which we found a problem of pre-formed plasma within the cone due to the prepulse in the LFEX pulse shape. We also found serious hard x-ray and electromagnetic pulse environment for plasma diagnostics when the target was irradiated by LFEX. In 2010, the second integrated experiment was successfully performed with improved pulse contrast in LFEX and improved plasma diagnostics, in which neutron enhancement exceeded the original experiment performed in 2002 [4].

Further tuning of LFEX and extended experiments are on going to accomplish the goal of FIREX-1, $5 \mathrm{keV}$. FIREX-2 is proposed to demonstrate the real ignition and fusion burn by FI scheme with 100-kJ class lasers.

\section{ACTIVATION OF LFEX LASER}

LFEX is a Nd:glass-based $1.053-\mu \mathrm{m}$ four-beam laser system. Its final output will be $10 \mathrm{~kJ}$ in a $0.5-20 \mathrm{ps}$ pulse. The full system has four $37 \times 37 \mathrm{~cm}$ square beams. A pulse from the oscillator is spectrally chirped and is amplified with three-stage OPCPA (optical parametric chirped pulse amplification) system, rod amplifiers, and a four-pass large-aperture disk amplifier which has been constructed beside the Gekko-XII laser as shown in Fig. 1 (left). The pulse is then compressed with large grating pulse-compression system down to $1 \mathrm{ps}$. The beams are focused with an off-axis parabolic mirror ( $\mathrm{f}=4000 \mathrm{~mm}$ ). Rear-end system is constructed within a large vacuum chamber located near the interaction chamber of Gekko-XII as shown in Fig. 1 (right). Beam synchronization of LFEX to GekkoXII is performed optically by using a common oscillator pulse for both lasers. 
Two beams among four were activated in 2010. The compressed pulse width for one beam was $1.2 \mathrm{ps}$ and the other was $1.3 \mathrm{ps}$. When two beams were overlapped, it was $1.5 \mathrm{ps}$. Pulse contrast ratio was substantially improved in 2010. Saturable absorbers and AOPF (amplified optical parametric fluorescence) quenchers [6] were introduced to the OPCPA stages to reduce the prepulse component down to a pulse contrast of $10^{-8}$.

Beam focal spot size was estimated from x-ray images of planar targets irradiated by LFEX beams. Observed focal spot was 30-60 $\mu \mathrm{m}$ in diameter, which is nearly twice the diffraction limit, resulting in an irradiation intensity of order of $1 \times 10^{19} \mathrm{~W} / \mathrm{cm}^{2}$ on target. The size was the same in cases of one beam and two beams overlapped on the target.

We limited the output energy so far to avoid the damage of the optics, particularly the compressor gratings, to be up to $700 \mathrm{~J} / \mathrm{beam}$ in $1 \mathrm{ps}$, for example.

\section{INTEGRATED EXPERIMENT OF FAST IGNITION}

\subsection{Experiment with Gekko-XII and LFEX lasers}

Implosion and heating experiments of Fast Ignition (FI) targets for FIREX-1 have been performed by operating both Gekko-XII and LFEX lasers. Typical laser and target parameters were as follows. GekkoXII laser for implosion: $0.53-\mu \mathrm{m}$ light with an energy of $1.5-4.5 \mathrm{~kJ}$ in total in a $1.5 \mathrm{~ns}$ nearly Gaussian pulse in 2009 as in 2002, and a nearly flat-top pulse in 2010 experiment, nine beams among twelve. LFEX laser for heating: $1.053-\mu \mathrm{m}$ light with an energy up to $1-2 \mathrm{~kJ}$ in $1-5 \mathrm{ps}$. The beam(s) were focused and injected into a cone attached to a shell target. Shell targets (CD: deuterated polystyrene): $500 \mu \mathrm{m}$ in diameter and $7 \mu \mathrm{m}$ in thickness. A 10-20 $\mu \mathrm{m}$ wall-thickness Au cone with an opening angle of 30 or 45 degrees. Outer surface of the Au cone was coated with 10- $\mu$ m-thick $\mathrm{CH}$ layer. Distance from the center of the shell to the cone tip was $50 \mu \mathrm{m}$.

Characteristics of the imploded and heated fuel plasma were observed by using a variety of plasma diagnostics. Dynamics of the imploded fuel plasma was observed with ultrafast x-ray spectroscopic imaging utilizing x-ray streak cameras [7-9] and x-ray framing cameras [10]. Fusion products were observed with detectors including a multi-channel single-hit neutron spectrometer, ultrafast liquid scintillator neutron detectors [11], filtered CR-39 detectors, and so forth. Hot electrons generated with LFEX beam irradiation were observed with electron spectrometers. In 2009 experiments, we had serious problems when we used LFEX laser. Laser intensity on the target was so high and the amount of the laser energy was so large that tremendous amounts of hard $\mathrm{x}$-rays $(\gamma$ rays) and electro-magnetic pulse (EMP) were generated, and the plasma diagnostics were in a very serious condition. In 2010 experiment, we have improved these plasma diagnostic instruments to be more compatible with such serious hard $\mathrm{x}$-ray and EMP harsh environment. High-performance shielding boxes were introduced to shield electronics and computers from intense EMP. Total reflection x-ray mirrors were coupled to $\mathrm{x}$-ray framing cameras to choose only thermal $\mathrm{x}$-ray images of the imploded core plasma. Time-resolved two-dimensional x-ray images of the imploded core plasma was obtained with Multi-Imaging X-ray Streak Camera [7] equipped with a hard-x-ray shielded photo cathode disk. Injection time of the heating beam relative to the $\mathrm{x}$-ray emission history from the imploded core was measured with this x-ray streak camera with an accuracy better than $+/-10$ ps by using non-imaged hard $x$-ray signals.

\subsection{Fast heating of the fuel plasma}

Figure 2 shows neutron yield obtained from imploded and heated fuel plasma with heating by 1 ps PW laser in 2002 [4], 1 ps and 5 ps LFEX beam in 2009, and 1.5 ps LFEX in 2010.

The neutron yield and ion temperature curves were calculated with a hydro simulation code assuming the initial condition of the imploded fuel plasma as well as the heating efficiencies. The hydro code used in the calculation is a one-fluid, two-temperature Euler-type hydrocode with radiation 

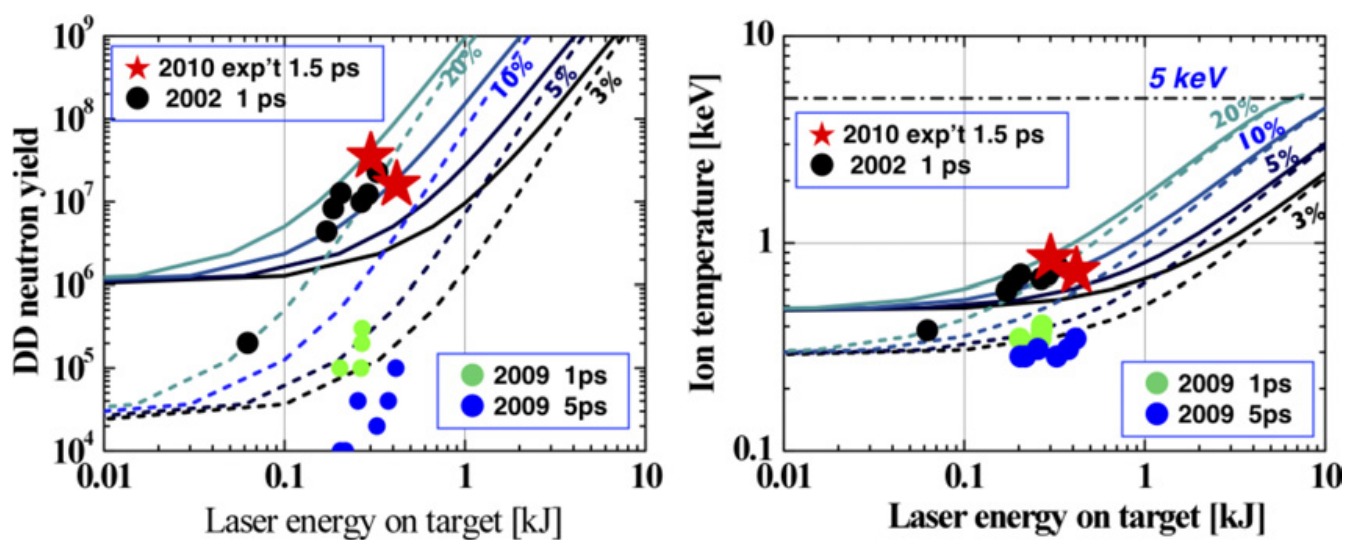

Figure 2. (Left) Neutron Yield obtained in 2010, 2009, and 2002 experiments. Curves are from simulations with assumed efficiencies. (Right) Estimated ion temperature for the same data.

and thermal transport and fusion reactions. Although the actual density of the core plasma was not yet experimentally measured, here in the simulations, the initial density profile before the fast heating was assumed as a typical one of the implosion at this level to be a Gaussian distribution with a central peak density of $100 \mathrm{~g} / \mathrm{cm}^{3}$, and a half density radius of $20 \mu \mathrm{m}$, resulting in a $\rho R=0.2 \mathrm{~g} / \mathrm{cm}^{2}$. Initial temperature of the core plasma in the simulation was assumed to be uniform and set to match the experimentally observed neutron yield without fast heating with the assumed density profile. The neutron yield without heating beam was $1 \times 10^{4}$ in 2002 and 2009 experiments, and $1 \times 10^{6}$ in 2010 experiment. Difference is due to different pulse shapes of the driving Gekko-XII laser for implosion. We assumed a uniform temperature-rise model in which heating was assumed to be sudden and to have uniform temperature distribution. Thermal energy due to heating was suddenly given to the electrons, and ions received energy from electrons via collisions. The hydrodynamics were calculated after the sudden heating, and the neutron production was accumulated. Rise in the ion temperature (figure 2, right) and the enhanced neutron yield (figure 2, left) were calculated for specific values of heating efficiency (ratio of thermal energy increase in the core plasma to heating laser energy on target), as indicated in Fig. 2. Dashed curves are for 2002 and 2009 data, and solid curves are for 2010 data.

In 2009 experiment, enhancement of the neutron yield compared with no heating case has been achieved by 1 ps or 5 ps heating. Note that a 30-degree cone was used for the 5 ps data, and a 45 -degree one for $1 \mathrm{ps}$. Although the result looks as if there is a strong dependence on the pulse width, it is not clear because other experimental conditions were not the same. The ion temperature estimated from $1 \mathrm{ps}$ and 5 ps data in 2009 indicated that the heating efficiency was only 3-5\% or less, which is much lower than $20-30 \%$ in the previous 2002 data.

Observed hot electrons in 2009 had higher energy spectrum of about $10 \mathrm{MeV}$ than the expected value, a few $\mathrm{MeV}$, required for efficient energy deposition in the present level of the expected fuel $\rho R, 100-300 \mathrm{mg} / \mathrm{cm}^{2}$. According to a separate measurement of the preformed plasma and simulation analyses, it was attributed to the preformed long-scalelength plasma created with a prepulse in the LFEX beam and confined in the cone. Prepulse level was significantly reduced in 2010 experiment to improve the heating efficiency.

Also we found that not only the DD fusion neutrons from the imploded and heated core but also neutrons due to $(\gamma, \mathrm{n})$ reactions and scattered $\gamma$ rays were detected by time-of-flight neutron detectors. Materials such as the vacuum chamber as well as the plasma diagnostic instruments were irradiated with intense hard $\mathrm{x}$-rays ( $\gamma$ rays) generated via Bremsstrahlung from the hot electrons in the target irradiated with LFEX beams. Signals of $(\gamma, \mathrm{n})$ neutrons and scattered $\gamma$ rays were analyzed by using Monte-Carlo 
simulations. Such obstacle signals were carefully eliminated and the net DD neutron signals were extracted to determine the enhanced DD neutron yield. The neutron yield was measured much more accurately in 2010.

In 2010 experiment, we have observed neutron enhancement up to $3.5 \times 10^{7}$ with total heating energy of $300 \mathrm{~J}$ on target, which is higher than the yield obtained in 2009 experiment and exceeded the previous data in 2002. Neutron enhancement was observed only when the heating laser was injected within $50 \mathrm{ps}$ around the time of the maximum $\mathrm{x}$-ray emission from the core. Estimated ion temperature is up to $0.8 \mathrm{keV}$. We found the estimated heating efficiency is at a level of 10-20\%. In the coming next experiment from 2012 , heating up to $2-3 \mathrm{keV}$ is expected with $2-3 \mathrm{~kJ}$ heating, and $5 \mathrm{keV}$ with $7-10 \mathrm{~kJ}$, if we can keep a heating efficiency of $20 \%$.

The heating efficiency depends on several parameters of the fast electron beam and its transport to the core. According to the simulations including Fokker-Planck transport [12] for an almost similar laser-plasma condition, the efficiencies without preformed plasma in the cone were estimated to be $48 \%$ from the heating laser to the fast electrons ( $39 \%$ to the electrons less than $10 \mathrm{MeV}$ ), $16 \%$ from the fast electrons to the core, resulting in the total efficiency to be about $8 \%$. The value obtained in 2010 experiment, $10-20 \%$, is close to or higher than that. It is also higher than the results obtained in the experiment with the OMEGA laser at University of Rochester, 3.5+/ - 1.0\% [13]. However, it is yet difficult to discuss the difference in detail to have a decisive view of the heating process, because there are many unknown factors in the electron beam parameters as well as the heating model. Further investigations are needed.

Data for only two target shots in 2010 are plotted in figure 2, although we made almost 40 integrated target shots. Unfortunately, the neutron signals were completely overwhelmed with after glow of the scintillator signal of intense $\mathrm{x}$-rays and other background signals in the target shots with the LFEX energy larger than $400 \mathrm{~J}$. Further improvement of the diagnostics is essential in the next experiments.

\section{CONCLUSIONS}

Enhancement of the neutron generation up to $3.5 \times 10^{7}$ due to fast heating of the imploded fuel plasma has been achieved in the reactivated integrated experiment of Fast Ignition with LFEX laser at ILE, Osaka. Results of 2002 experiment were reconfirmed with much more accurate plasma diagnostics in 2010. Estimated heating efficiency in 2010 assuming the uniform temperature-rise model is 10-20\%.

Further tuning of LFEX is underway to extend the FI integrated experiments. Full four beams will be activated in 2011-2012. 5-keV heating is expected with full output of LFEX. Further improvement in the heating efficiency with advanced target concept is expected to result in achievement of $5 \mathrm{keV}$ heating with even smaller energy of the heating beam.

The authors would like to thank Gekko-XII and LFEX laser operation crew, target fabrication group, plasma diagnostics group, and computer operation staffs for their great contributions to this work. This work was supported by Interactive Coordinated Research Program with the National Institute for Fusion Science, Japan (NIFS11KUGK046).

\section{References}

[1] T. Yamanaka (1983) (unpublished)

[2] N. G. Basov, S. Yu. Gus'kov, and L. P. Feoktistov, J. Sov. Laser Res. 13, 396 (1992)

[3] M. Tabak, J. Hammer, M. E. Glinsky, W. L. Kruer, S. C. Wilks, J. Woodworth, E. M. Campbell, M. D. Perry, R. J. Mason, Phys Plasmas 1, 1626 (1994) 
[4] R. Kodama, P. A. Norreys, K. Mima, A. E. Dangor, R. G. Evance, H. Fujita, Y. Kitagawa, K. Kurshelnick, T. Miyakoshi, N. Miyanaga, et al., Nature 412, 798 (2001); R. Kodama, H. Shiraga, K. Shigemori, Y. Toyama, S. Fujioka, H. Azechi, H. Fujita, H. Habara, T. Hall, Y. Izawa, et al., ibid, 418, 933 (2002)

[5] H. Azechi, K. Mima, Y. Fujimoto, S. Fujioka, H. Homma, M. Isobe, A. Iwamoto, T. Jitsuno, T. Johzaki, R. Kodama, M. Koga, K. Kondo, J. Kawanaka, T. Mito, N. Miyanaga, O. Motojima, M. Murakami, H. Nagatomo, K. Nagai, M. Nakai, T. Nakamura, K. Nishihara, H. Nishimura, T. Norimatsu, T. Ozaki, H. Sakagami, Y. Sakawa, N. Sarukura, K. Shigemori, T. Shimizu, H. Shiraga, A. Sunahara, T. Taguchi, K. A. Tanaka, K. Tsubakimoto, Nuclear Fusion 49, 104024 (2009)

[6] K. Kondo, H. Maeda, Y. Hama, S. Morita, A. Zoubir, R. Kodama, K. A. Tanaka, Y. Kitagawa, and Y. Izawa, J. Opt. Soc. Am. B 23, 231 (2006)

[7] J. Zhong, H. Shiraga, and H. Azechi, Rev. Sci. Instrum. 79, 10E907 (2008)

[8] H. Shiraga, M. Lee, N. Mahigashi, S. Fujioka, and H. Azechi, Rev. Sci. Instrum. 79, 10E920 (2008)

[9] M. Tanabe, T. Fujiwara, S. Fujioka, H. Nishimura, H. Shiraga, H. Azechi, and K. Mima, Rev. Sci. Instrum. 79, 10E908 (2008)

[10] M. Koga, T. Fujiwara, T. Sakaiya, M. Lee, K. Shigemori, H. Shiraga, and H. Azechi, Rev. Sci. Instrum. 79, 10E909 (2008)

[11] T. Nagai, Y. Ioka, A. Hasegawa, K. Wada, S. Takaoku, M. Takata, K. Noritake, Y. Minami, K. Watanabe, K. Yamanoi, Y. Arikawa, H. Hosoda, H. Nakamura, T. Watari, M. Cadatal-Raduban, M. Koga, T. Shimizu, N. Sarukura, H. Shiraga, M. Nakai, T. Norimatsu, and H. Azechi, Jpn. J. Appl. Phys 50, 080208 (2011)

[12] T. Johzaki, H. Nagatomo, A. Sunahara, H-B. Cai, H. Sakagami, Y. Nakao, and K. Mima, Nuclear Fusion 51, 073022 (2011)

[13] W. Theobald, A. A. Solodov, C. Stoeckl, K. S. Andersen, et al., Phys. Plasmas 18, 056305 (2011) 